\title{
VISUAL CONTRACTS A Way to Reason About States and Cardinalities in IT System Specifications
}

\author{
José D. De la Cruz, Lam-Son Lê, Alain Wegmann \\ School of Computer and Communications Sciences, \\ Ecole Polytechnique Fédérale de Lausanne, CH-1015 Lausanne, Switzerland
}

Keywords: Visual Languages, UML, Specification, Conceptual Modeling, Hierarchical Systems, Model-Checking.

\begin{abstract}
Visual modeling languages propose specialized diagrams to represent behaviour and concepts necessary to specify IT systems. As a result, to understand a specification, the modeller needs to analyze these two types of diagrams and, often, additional statements that make explicit the relationships between them. In this paper, we define a visual contract notation that integrates behaviour and concepts. Thanks to this notation, the modeler can specify, within one diagram, an action and its effects on the specified IT system. The notation semantics is illustrated by a mapping to Alloy, a light weight formal language.
\end{abstract}

\section{INTRODUCTION}

The creation of the specification of an IT system is a complex task that requires the division of the specification into smaller, partial descriptions (e.g. conceptual class diagram, state machine diagram, etc.) portraying each a perspective of the system. In this paper, we focus on the division between conceptual and behavioural diagrams in graphical specifications. When such separation is made, four main drawbacks appear: First, there are not precise relationships among the different diagrams. Secondly, each kind of diagram is expressed using its own language. Third, the constraints are generally expressed in yet another language. Fourth, the system specification cannot be easily validated.

Our concern is how to "glue back" the partial system descriptions together in order to obtain a holistic view of the system that can be validated. We propose a notation that integrates conceptual specification together with behavioural specification. The goals are to facilitate a) the reasoning of the modellers in the terms of actions/services, and $b$ ) to enable the model checking of the specification. We map our visual notation onto the formal language Alloy (Jackson, 2002) in order to validate its soundness.

Throughout the paper we show the example of an IT system (BoardingITSystem) that controls the boarding process of passengers to a plane. Initially, the plane is empty but the passengers have already checked-in at the company ticket counter. Then the system processes the requests from passengers that are ready for going onboard: passengers' boarding passes are processed one by one until the plane's maximum capacity is reached. As a safety rule, none of the passengers can board or disembark more than once. Figure 1 illustrates this example using the UML notation (OMG, 2005).

Section 2 defines visual contracts and their semantics (in Alloy). Section 3 presents the BoardingITSystem example using our visual contract notation. Section 4 is the state of the art.

\section{VISUAL CONTRACT}

We define Visual Contract (VC) as the visual model that represents both the pre and the post conditions for an action as well as the changes between the two. Our visual contract notation is developed in the context of SEAM (Systemic Enterprise Architecture Methodology), a method designed for reasoning about business and IT alignment (Wegmann et al, 2005).

In our approach, we model the behaviour together with the state of the systems. Our modelling ontology is based on RM-ODP(ISO/IEC \& ITU-T, 1998) and on our formalization of it (Le \& Wegmann, 2005). 

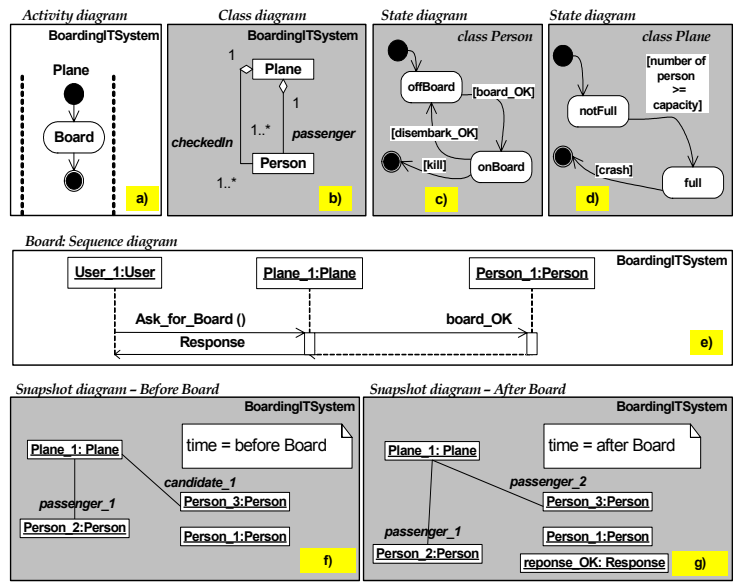

Figure 1: A partial UML specification for activity Board of system BoardingITSystem.

A system is modelled as a RM-ODP object that we call working object. Working objects can be specified as whole or as composite. A working object as whole is described atomically. Only the externally visible behaviour is described using a model-based description (Schätz et al, 2002). A working object as composite is described as a set of component working objects that collaborate together. In visual contracts, we consider only working objects as whole.

Working objects as whole are described in terms of information objects, set associations and localized actions.

\subsection{Information Objects}

An Information Object (IO for short) captures the type and the possible states of the concepts necessary to describe the observed system.

For example, Figure 2 represents the IO Person that captures the information of a person in the real world. The attribute Boarded captures the state of a Person in relation with the Plane she wants to board. All IOs include an identity attribute Id.

\begin{tabular}{|l|l|}
\hline \multicolumn{2}{|c|}{ Person } \\
\hline Boarded & offBoard \\
\hline Id & onBoard \\
\hline
\end{tabular}

Figure 2: Information Object representation.

\subsection{Set-Associations}

We claim that when reasoning with graphical models our minds use instances implicitly.

Set-associations (SA) captures information about these instances. As set-associations relate IOs, it means that these instances exist within a context.
For example a set-association between a Plane and a Person can represent a person that is either offBoard or onBoard. This is illustrated in Figure 3. For practical reasons, we avoid drawing the single instances (extensional form, like in the object diagrams of UML), and we use instead cardinality and state information of the set of instances. We call this the intensional form.

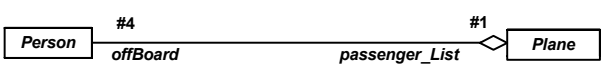

Figure 3: SEAM representation of 4 passengers using instance cardinalities.

As illustrated in Figure 3, a SA requires a name (passenger List), a referring instance cardinality $(\# 1)$, a set of referred instances cardinality (\#4), and a state (offBoard).

\subsection{Actions}

As illustrated in Figure 1, an action can be modelled in UML by a set of quasi-orthogonal diagrams (that can be related to each other via OCL). In contrast, our visual contract diagrammatically describes in a single diagram an action and its effects.

An action specifies the effects of system's reactions to a set of events for a given system state. When we consider a given action $\mathrm{A}(\mathcal{A})$, we describe the set of predicates for the initial conditions $(\mathscr{P})$ that will guarantee that the final state (Q) is reached, as expressed in (Hoare, 1969):

$$
\{P\} \mathcal{A}\{Q]
$$

Since state information of objects is represented at the set associations, the change of global state is equivalent to the sum of changes in set associations: changes of cardinality and of state.

Figure 4 and Figure 5 represent diagrammatically changes due to actions op1 and op2, respectively.

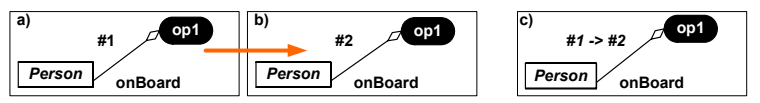

Figure 4: Action changes cardinality of set-associations. On the left side the initial (a) and final (b) conditions. In (c) SEAM notation for representing changes.

In Figure 4. $a$ there is only a single instance of type Person. After op1, there are two instances of the same IO, as shown in Figure 4.b. The state of the instances does not change. The Figure $4 \mathrm{c}$ is the SEAM equivalent to the evolution from 4. $a$ to $4 . b$.

In Figure 5, we illustrate a state change (without cardinality changes). Initially, the SA selected includes one instance of IO Person that is offBoard 
(5.a). At the end, this same person is onboard (5.b). Figure 5.c represents the evolution from 5.a to $5 . b$.

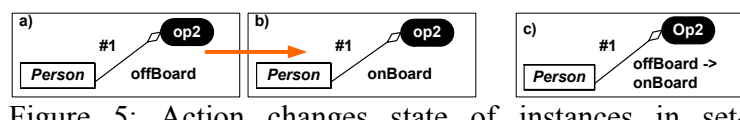

Figure 5: Action changes state of instances in setassociations.

Figures 4.c. and 5.c introduce the «change» operator $(\rightarrow)$, central in our approach. As a corollary, we may say that what is not shown as changing in the specification of an action is considered to remain unchanged.

\subsection{Other Modeling Constructs}

We can reason about systems because we can see how actions change the instances. Therefore, it is fundamental to model the lifecycles of the instances (to understand which instances exist and when). A context of existence of an instance is the temporal frame where an instance or a set of instances exist. Our semantics for the context of existence is similar to UML composition/ aggregation, so we will use the UML notation (black/hollow diamond).

Given that all instances exist in a context, there is a "first context" for each system. It is the model element Myself. This element represents the "root" of all behavioural and conceptual information describing the system. The double nature of this model element is represented by a symbol that combines an IO and an action:

\section{Myself}

We also define a special kind of information object: the parameter. Parameters are necessary to represent the communication through the boundary of the system or through action boundaries. The stereotype can be «Par In» or «Par Out» depending on whether it is an input or an output parameter.

\section{EXAMPLE: THE PLANE BOARDING CONTROL SYSTEM}

In this Section, we present the visual contract that corresponds to Figure 1. First we introduce the necessary information objects (Section 3.1). We then define the operations Init and CheckIn (Section 3.2) and then Board (Section 3.3). Only a normal, correct case of behaviour is shown in this paper.
For each diagram we present, we define the Alloy equivalent. Alloy is a light weight modelcheckable specification language that we use to define the semantics of our notation.

\subsection{Definition of Information Objects}

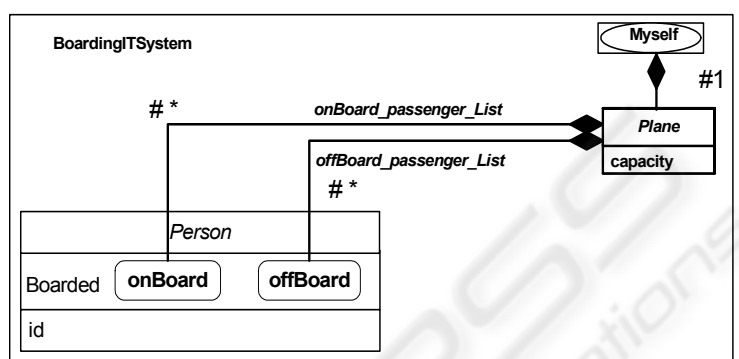

Figure 6: SEAM notation for data definitions for the BoardingITSystem.

For sake of simplicity, the system BoardingITSystem knows one Plane only. SEAM's representation, shown in Figure 6, can be read as follows: In the system (i.e. Myself), there is the knowledge of one Plane with a capacity. This Plane has two passenger_List that can contain multiple passengers each; one is for the passengers that have checked-in (offBoard) and the other one for the ones that have effectively boarded the Plane (onBoard).

The Alloy equivalent is shown in figure 7.

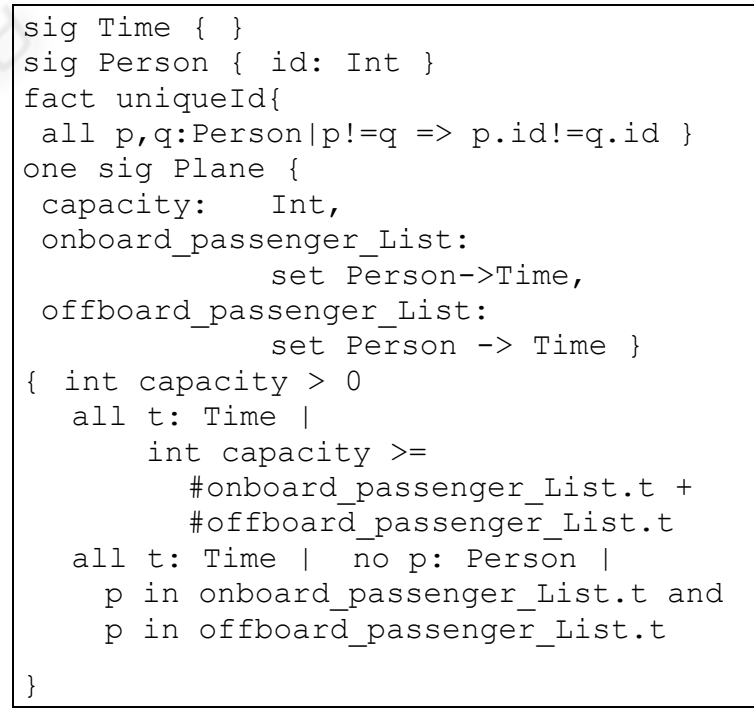

Figure 7: Partial Alloy specification for action Board.

The Alloy can be read as follows: a set of ordered time points are defined (sig Time); a set of Person are defined (sig Person) with a unique 
identifier (fact uniqueID). We define also a Plane that has a capacity and 2 lists: onboard Passenger List and offBoard_Pass enger_List. These lists include a relation between a person and a time point (necessary to simulate the execution sequence). Some invariants are defined in the plane: the capacity is never exceeded, and nobody can be in both lists at the same time.

\subsection{Operations Init \& CheckIn}

Figure 8 shows the SEAM visual contract for operation Init. It states that the number of Person in both passenger_List (offBoard, onBoard) is set to zero.

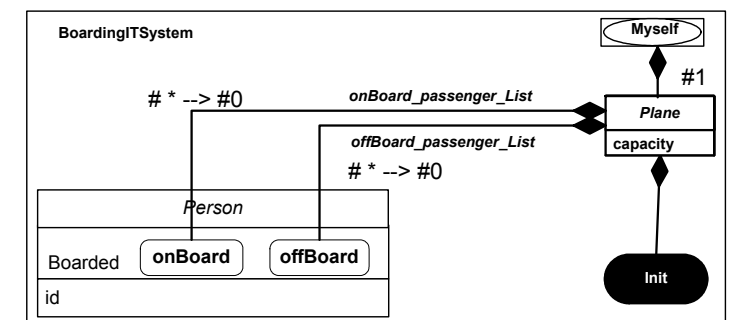

Figure 8: The SEAM contract of Init: the cardinality of the passenger_List SA changes.

The cardinality of each set-association that links passenger_List to Person goes from some initial value (any, symbolized by the character '*') to 0 . In the practice, this means erasing all instances of Person linked to both passenger_List (offBoard, onboard).

The action checkIn, not presented in this paper, assigns instances of Person to the offBoard passenger List.

\subsection{Operation Board}

The specification of action Board is the following: "The plane preconditions are: $a$ ) an input parameter represents the identifier of the person that desires to go on board, $b$ ) this person has already checked-in, and c) the number of people onboard has not reached the maximum capacity of the plane. The post condition is that the person is now onboard. In addition, the system emits a message confirming the entry of the person into the plane".

Before creating the Visual Contract for action Board, we illustrate the action by making two snapshots: one before and one after the operation Board. The situation would be as shown in Figures 9 and 10 , respectively.

In the precondition, there is an instance of
Id_Person that is considered as valid. The valid condition is defined by a constraint in the diagram: the Id_Person should correspond to the id of only one Person that has already checked-in (she is in the offBoard_passenger_List).

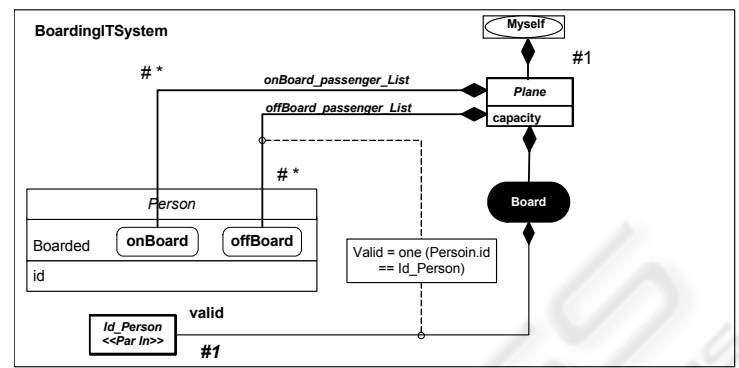

Figure 9: Precondition for action Board.

During the action Board, the parameter Id_Person is validated and the corresponding instance of Person in the offBoard passenger_List is referenced by the action via the SA selected.

In the post condition, Figure 10, the selected instance of Person will be transferred to the onBoard_passenger_List (supposedly the one that has been admitted in the precondition); the cardinality change in the corresponding SA symbolizes this. Simultaneously, the offBoard passenger List is decremented by one. Finally, a Response parameter is emitted, indicating the success of the operation (represented by the SA Greetings_Response).

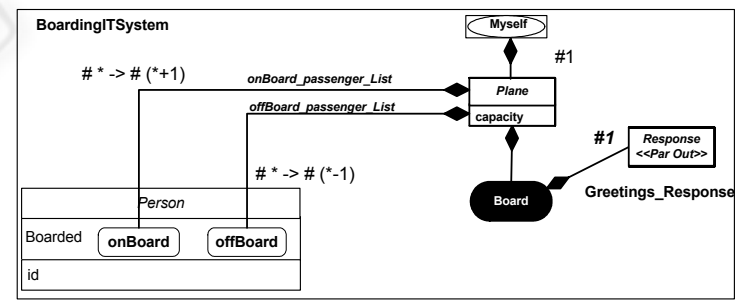

Figure 10: Post condition of action Board.

The whole visual contract is shown in Figure 11. Here we make explicit the changes and the instances involved, in order to avoid misunderstandings. The instance of the offBoard_passenger_List that corresponds to the Id Person is represented by the SA selected, that exists in the context of action Board. Remark that this temporary information does appear neither in the pre nor in the post condition.

Notice also that the constraint regarding the Plane capacity has become a guard for a transfer of the instance selected of IO Person from offBoard_passenger_List onto the onBoard passenger_List. 


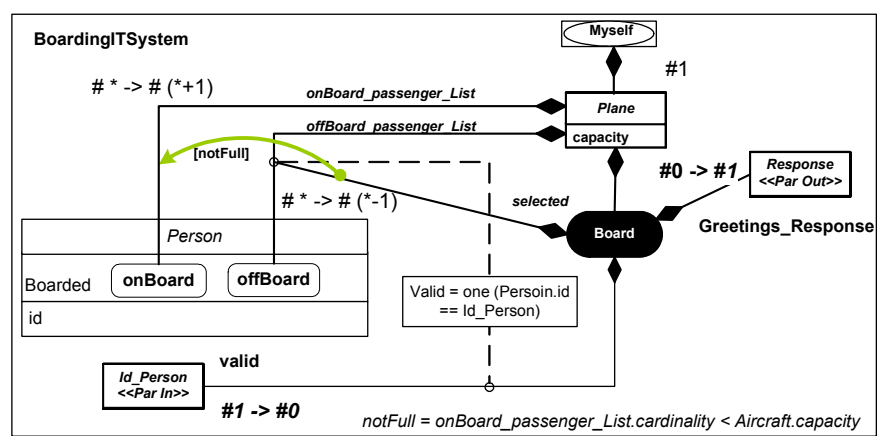

Figure 11: Visual contract for action Board. It illustrates the «change» operator.

The Figure 12 illustrates the evolution of state of the IT system during the contract execution. It is the result of the execution of the Alloy code presented below. It shows a scenario where the plane has two people (Person0, Person1) in the offBoard passenger_List before the action execution (at Time 0). One passenger (Person1) actually boards the plane, as can be seen in the nodes that represent the state after the action execution (at Time1). The person Persono has changed to the onBoard_passenger_List.

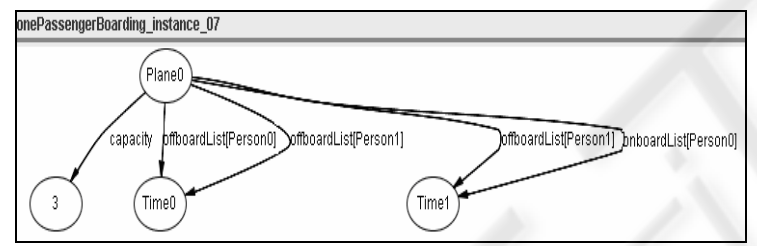

Figure 12: Result of simulating the action Board in the Alloy analyzer. Time 0 and Time 0 are the moment before and after the action takes place.

\section{RELATED WORK}

UML (OMG, 2005) is the industry standard in object-oriented analysis and design. UML creating heterogeneous models made up of different diagrams and notations. In our approach, we try to minimize the number of diagrams to one by representing conceptual and behavioural specifications together. Our syntax is comparable to UML -in order to improve the usability - however semantics are totally dissimilar. We can say that VCs are complementary to UML diagrams.

One of the challenges we face is how to express the relationships between instance and types in conceptual modelling. UML proposes the use of separate diagrams: class and object (OMG, 2005). Heckel (Heckel \& Sauer, 2001) deals with types and instances in collaboration diagrams. While these approaches represent static information, our goal is to represent how actions change information.

The use of contracts allowed validating implementations of object-oriented designs (Helm et al; 1990; Meyer, 1992). The use of contracts to specify actions, interactions, use cases, and activities is presented in (D'Souza \& Cameron Wills, 1998; Rik Eshuis, 2001; Stevens, 2001; Wirfs-Brock et al, 1990). However, most of the work has been done mostly using OCL and textual logics. Only recently, two research groups (Lohmann et al, 2005; De la Cruz et al, 2005) have proposed independently the concept of "visual contracts". The first focuses on automatic generation of code that reinforces the compliance of the applications of the IT system with the constraints and business rules whereas the latter focuses on conceptual modelling and IT system specification.

The graph transformation (GT) languages like QVT (OMG, 2005) , VPM (Varro \& Pataricza, 2003) and the one proposed by Heckel (Heckel \& Sauer, 2001) are syntactically similar to our contracts because $a$ ) we use UML-like notation and b) the contracts are also executed according to a pattern-matching oriented philosophy. However, they use OCL and are used mostly for transformation among languages -e.g. class diagrams to java code, statecharts to sequence diagrams, etc. - at the metamodel level. They are not used as languages for specification but for extraction/transformation from models that should generate object-oriented code. Their primary goal is to guarantee well-formedness and translatability, and not to support reasoning about actions/services and system evolution. Besides, our approach is declarative whereas many of the GT languages are mostly operational/ imperative. Our approach is complementary to these UML-based initiatives. 


\section{CONCLUSIONS AND FUTURE WORK}

In order to define visual contracts, we extend the traditional interpretation of the association and we integrate diagrams that are usually considered as separate. We define the concept of set associations that capture the existence of instances of concepts in a given context. We use these set associations to relate information objects (our term for concepts) to information objects but also actions to information objects. To represent the pre and the post condition on a single diagram, we define a new graphical symbol to express the change of cardinality or state.

Our visual contracts addressed the four issues raised by the separation of conceptual and behavioral diagrams for modeling actions. First, differente descriptions were put along in a single diagram; our visual contracts include structure, state, communication \& synchronization, action/activity and constraint information. Secondly, the proprietary languages of each notation were harmonized with each other by our semantics. Third, constraints were illustrated as logic guards for changes in the visual contracts. Fourth, the diagram themselves can be used to reason about the system actions.

We also reconcile and integrate the formal methods that allow us model-checking and simulating our models. The mapping between the visual contracts and a model-checkable language as Alloy is useful to validate the specification model before a real/complex implementation is done.

Our future work includes testing and improving the usability of the notation, developing tool support that translates automatically the visual contract into the Alloy code, and the integration of this feature into our CAD tool (Le \& Wegmann, 2005), as well as formalizing the model-checking capability. We plan also to build a UML to SEAM translation tool.

\section{REFERENCES}

De la Cruz, J. D., Wegmann, A., \& Regev, G. (2005). Expressing Systemic Contexts in Visual Models of System Specifications. In T. Bui \& A. Gachet (Eds.), Proceedings of the Workshop on Context Modeling and Decision Support, CONTEXT05 Workshops (Vol. CEUR 144). Retrieved October 31, 2005, from http://CEUR-WS.org/Vol144/04_deLaCruz.pdf

D'Souza, D. F., \& Cameron Wills, A. (1998). Objects, components, and frameworks with UML: The Catalysis approach (1 ed.): Addison Wesley Longman, Inc.
Heckel, R., \& Sauer, S. (2001). Strengthening UML Collaboration Diagrams by State Transformations. Paper presented at the FASE 2001, Genova, Italy.

Helm, R., Holland, I. M., \& Gangopadhyay, D. (1990). Contracts: Specifying Behavioural Compositions in Object-Oriented Systems. Paper presented at the OOPSLA/ECOOP 1990, Ottawa, Canada.

Hoare, C. A. R. (1969). An Axiomatic Basis for Computer Programming. Communications of the ACM (CACM), 12(10), 576-580.

ISO/IEC, \& ITU-T. (1998). Recommendation X.901, X.902, X.903, X.904, "Open Distributed Processing Reference Model" (Recommendation): ISO and ITU$\mathrm{T}$.

Jackson, D. (2002). Alloy: a lightweight object modelling notation. ACM Trans. Softw. Eng. Methodol., 11(2), 256-290.

Le, L. S., \& Wegmann, A. (2005). Definition of an ObjectOriented Modeling Language for Enterprise Architecture. Paper presented at the HICSS'05, Hawaii, USA.

Lohmann, M., Sauer, S., \& Engels, G. (2005). Executable Visual Contracts. Paper presented at the IEEE VL/HCC'05, Dallas, Texas, USA.

Meyer, B. (1992, Oct.). Applying "Design by Contract". IEEE Computer, 25, 40-51.

OMG. (2005). Unified Modeling Language (UML), from www.omg.org

Rik Eshuis, R. W. (2001). A Real-Time Execution Semantics for UML Activity Diagrams. Paper presented at the FASE 2001, Genova, Italy.

Schätz, B., Pretschner, A., Huber, F., \& Philipps, J. (2002). Model-based development of embedded systems. In J.-M. Bruel \& Z. Bellahsene (Eds.), Advances in Object-Oriented Information Systems, OOIS 2002 Workshops (Vol. LNCS 2426, pp. 298312). Montpellier, France: Springer.

Stevens, P. (2001). On Use Cases and Their Relationships in the Unified Modelling Language. Paper presented at the FASE 2001, Genova, Italy.

Varró, D., \& Pataricza, A. (2003, Aug.). VPM: A visual, precise and multilevel metamodeling framework for describing mathematical domains and UML. Software and Systems Modeling, 2(3), 187-210.

Wegmann, A., Balabko, P., Le, L.-S., Regev, G., \& Rychkova, I. (2005). A Method and Tool for BusinessIT Alignment in Enterprise Architecture. Paper presented at the CAiSE'05, Porto, Portugal.

Wirfs-Brock, R., Wilkerson, B., \& Wiener, L. (1990). Designing Object-Oriented Software (1 $\begin{array}{ll}1 & \mathrm{ed} .) \text {. }\end{array}$ Englewood Cliffs: Prentice Hall. 